\title{
An Advanced Platform for Thermodynamics Education. Part one: Small Density Pure Real Gases
}

\author{
B.I. Sedunov \\ Russian New University (ROSNOU) \\ 22 Radio st., Moscow, Russia \\ E-mail: sedunov.b@gmail.com
}

Received 30 March 2020, Accepted 11 August 2020

\begin{abstract}
The paper presents an advanced platform for thermodynamics education - the equilibrium pure real gas system, which has been deeply investigated experimentally and theoretically by researchers from all over the World. But in spite of a huge amount of extra precise experimental data, the nature of real gases and clusters in them is still poorly understood. The clusters are considered now as a new state of matter. To study them is both challenging and educative. We use a wonderful feature of pure real gases: the chemical potential for all basic particles in a gas is universal for all clusters. It permits us to find the monomer fraction density $D_{m}$ from an experimental pressure dependence of the total density. This variable has proven to be the key to properties of clusters and molecular interactions. The advanced platform is more informative for thermodynamics education than the widely used ideal gas model, which ignores molecular interactions. It provides a new vision of molecular interactions in clusters and the entropy in pure real gases. Moving step-by-step from ideal to denser gases students may better understand the complex nature of condensation. Here we start from small density gases.
\end{abstract}

Keywords: real gas; thermophysical properties; clusters; molecular interactions; entropy; chemical potential.

\section{Aims and Scope}

The general thermodynamics is very hard for comprehension by students; therefore, for teachers and students specific platforms are needed, where the thermodynamics laws appear as rather obvious and intuitively understandable. The general thermodynamics of equilibrium systems benefits from its applications to specific platforms, demonstrating its laws and providing a further development for its basic principles. Basing on different platforms we comprehend the general thermodynamics from multiple points of view.

The real gas is far from being a simple system: it consists of multiple and diversified cluster fractions. Clusters are aggregates of gaseous particles, bound by weak intermolecular forces. Clusters and cluster fractions are fundamental elements of a real gas. The molecular interactions [1, 2] in real gases obey some special features, which prevent from a direct application of the chemical bond model to the clusters and cluster fractions.

The goal of this paper is to demonstrate the unknown before relations for the pure real gas platform, which is abundant of precise experimental data. The thermodynamics of real gases course should follow the general thermodynamics study. The computer aided analysis of precise experimental thermophysical data for pure real gases may provide students a clear and understandable introduction to the statistical physics.

Some bright examples of thermodynamics applications to specific platforms, widely used in thermodynamics education, are listed down here. These platforms are characterized by specific conditions for a system under investigation:
Table 1. The platforms and their features useful for thermodynamics education

\begin{tabular}{|c|c|}
\hline Plat & $\begin{array}{l}\text { The features useful for } \\
\text { thermodynamics education }\end{array}$ \\
\hline $\begin{array}{l}\text { The pure ideal gas } \\
\text { model }\end{array}$ & $\begin{array}{l}\text { The Universal gas law; } \\
\text { The understandable, educative } \\
\text { statistics. }\end{array}$ \\
\hline $\begin{array}{l}\text { The mixture of ideal } \\
\text { gases }\end{array}$ & $\begin{array}{l}\text { law for total } P \text { and } \\
\text { ssures }\end{array}$ \\
\hline $\begin{array}{l}\text { The equilibrium } \\
\text { chemical system }\end{array}$ & $\begin{array}{l}\text { The law of Mass action; } \\
\text { The Boltzmann law for } \\
\text { equilibrium constants }\end{array}$ \\
\hline adiabatic & The consta \\
\hline $\begin{array}{l}\text { The Joule-Thomson } \\
\text { process }\end{array}$ & $\mathrm{T}$ \\
\hline The & de equation for \\
\hline $\begin{array}{r}\text { spin } \\
\text { hetic system }\end{array}$ & The \\
\hline $\begin{array}{l}\text { The semiconductors } \\
\text { in a thermal } \\
\text { equilibrium }\end{array}$ & $\begin{array}{l}\text { The universal Fermi level for } \\
\text { electron and hole systems }\end{array}$ \\
\hline $\begin{array}{l}\text { The vapor-liquid } \\
\text { equilibrium system }\end{array}$ & $\begin{array}{l}\text { The Raoult's law, } \\
\text { The Dalton's law, } \\
\text { The Henry's law; } \\
\text { The Gibbs-Duhem relation; } \\
\text { The Clausius-Clapeyron relation }\end{array}$ \\
\hline $\begin{array}{l}\text { The pure fluid a } \\
\text { critical point }\end{array}$ & $\begin{array}{l}\text { The critical } \\
\text { phenomenon }\end{array}$ \\
\hline
\end{tabular}


This list may be proceeded further, but it already clearly shows the importance of specific platforms for comprehensible thermodynamics education.

The listed above thermodynamics laws may also help to better understand the real gas nature.

This research provides the next valuable lessons:

The accumulated in the whole World and available from online databases, such as the NIST Webbook [3], huge amount of extra precise experimental data on thermophysical properties of equilibrium pure real gases and denser fluids, may be used for an extraction of still unknown molecular interaction and clusters' properties from them.

The clusters' properties extraction from experimental data is a very educative process, which requires a new type of cluster expansion instead of the virial expansions.

For pure real gases cognition an advanced system of thermodynamics laws may be built.

To express this system of laws and to use it in the educational process an additional set of terms and functions should be introduced, as for any new branch of science.

Thermophysical properties of real gases are greately determined by clusters, which types and concentrations strongly depend on temperature $T$ and molar density $D$ of a gas. The cluster physics in gases builds the basis for thermodynamics of denser pure fluids. This research discovers the continuous transformation of types, dimensions and concentrations of clusters in gases basing on the interactive computer aided analysis of extra precise thermophysical data from the NIST Webbook [3].

It may sound strange, but now we understand better the galactic structure rather than the clusters in gases. Lynn Yarris from Lawrence Berkeley National Laboratory named clusters as a new state of matter [4]. An explosion of interest to clusters now is stimulated by their utilization as seeds for nanoparticles nucleation [5-10]. But small interparticle bond energy, as compared to the thermal agitation energy, makes the clusters' nature cognition difficult. Thermal agitation energy makes the clusters' physics cognition difficult. It might be better to say: 'clusters are a unknown yet state of matter'.

The pure real gas consists of cluster fractions with different numbers $n$ of basic particles in clusters. At $n=1$ we have the Monomer fraction. In spite of an extra short lifetime for clusters, they are free moving particles with the Dalton's law for their partial and total pressures. The clusters may exist in various isomer forms, with different bond energies, as it is shown at the Figure 1.

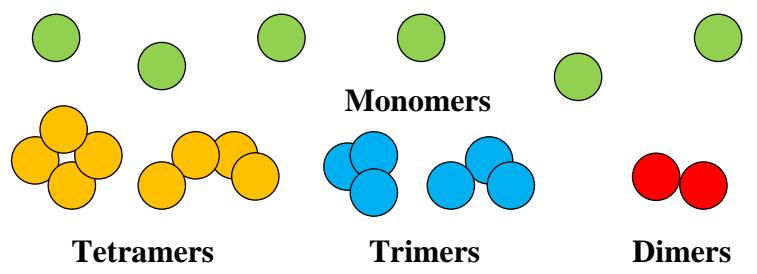

Figure 1. The Monomers and small Cluster isomers in a small density pure real gas.
The Figure 1 demonstrates the densely and loosely packed isomers of tetramers and trimers, possessing different bond energies. The isomers' bond energies may be found from the temperature dependence of the corresponding series expansion coefficients for potential energy density. It is very educative to estimate the isomers' bond energies for different gases.

Multiple earlier attempts to penetrate in the mystery of clusters [11-13] could not bring a comprehensive knowledge of the cluster nature. The key to clusters' properties extraction from precise experimental thermophysical data appeared only in 2007 with the monomer fraction density (MFD) $D_{m}$ introduction to the thermodynamics terms and functions family [14]. This idea had been developed further by the author in 2008 in the paper for International Journal of Thermodynamics [15].

The knowledge of the monomer fraction density $D_{m}$ in pure real gases opened the way to a cluster expansion by degrees of the monomer fraction density $D_{m}$. This new type of a cluster expansion has a clear statistical nature, corresponds to the Mass action law [16], and for this reason may be named as the canonical cluster expansion. Basing on the canonical cluster expansion the author has developed the interactive computer aided methods for thermophysical data analysis $[17,18]$. A high precision of the NIST Webbook [3] data, given with 12 digits, provides a satisfactory solution of the inverse problem [19] for hidden clusters' properties cognition.

\section{A new platform for thermodynamics education and research}

Pure real gases present an advanced platform for thermodynamics education [20] and research as the next step from the widely used ideal gas model, which ignores molecular interactions, being the most essential factors for the cluster and condensed matter physics. While the ideal gas model benefits from the molecular simulation methods, for the real gas nature cognition and comprehension the computer aided analysis of precise experimental thermophysical data has proven to be much more informative.

\subsection{The way to thermodynamics of fluids cognition and better comprehension}

We suggest the next strategy for pure fluids' structure investigation and teaching:

- The start from diluted pure real gases;

- $\quad$ Step-by-step movement from ideal gases through real gases to denser fluids;

- Basing on the precise experimental thermophysical data for pure fluids, such as the data from the NIST Webbook [3];

- Basing on the new informative variables, such as the monomer fraction density $D_{m}$, the density of free moving particles $D_{p}=P / R T$ and the fluid molar potential energy $U=E(T, P)-E(T, 0)$, where $E(T, P)$ is the molar Internal energy at a pressure $P$.

- $\quad$ Basing on the new type of cluster expansion with the Monomer fraction density $D_{m}$, as an argument for series expansions of different thermodynamics functions, to extract from precise experimental thermophysical data the properties of clusters and cluster fractions and understand better the molecular interactions nature. 
- The extension of the canonical cluster expansion methods for high densities to pave the way to better understanding of the gas-to-liquid transformation.

- $\quad$ Basing on the Cagniard de la Tour [21] and Thomas Andrews [22 - 24] conclusions of a continuous transformation from gaseous to liquid forms of matter to comprehend the supercritical fluids nature.

The earlier attempts [25-27] to understand the clusters' nature basing on the virial expansion of the real gas pressure $P$ by the total molar density $D$ failed [28]. Now we can explain why: the total density, as a sum of partial densities of basic particles in different cluster fractions, is not a correct physical argument for cluster expansions. Only the Monomer fraction density $D_{m}$ may serve as an argument for the cluster expansion to correspond to the law of Mass action [16]. It is useful for students to apply the canonical cluster expansion for different pure real gases. This training may help them to understand better the role of the Mass action law in statistical physics.

\subsection{New terms and variables for pure fluids}

The pure fluids structure cognition benefits from new terms and variables introduction:

- Basic particles - the particles of a pure fluid, corresponding to its chemical nature. Their introduction provides a new point of view on the fluid density $D$, which may be named as the total molar density of basic particles;

- Monomers - the basic particles, temporarily non interacting with other particles;

- $\quad$ The monomer fraction density [15] $D_{m}-$ the molar density of the monomer fraction;

- $\quad$ Free moving particles - monomers and clusters;

- Cluster isomer - the structural modification of an $n$-particle cluster;

- Chain cluster - the cluster isomer possessing no more than 2 bonds for a particle;

- The virtual cluster - the aggregate created by particles collision without their bonding;

- $\quad$ Partial density $D_{n}$ and partial pressure $P_{n}$ of the n-particle cluster fraction;

- $\quad$ The free moving particles density $D_{p}=P / R T-$ the sum of the monomers' and clusters' partial molar densities: $D_{p}=D_{m}+\Sigma D_{n}, \quad n=2,3, \ldots$

- The real gas potential energy $U=E(T, P)-$ $E(T, 0)[17]$ - the Excess molar Internal energy;

- The real gas potential enthalpy $H_{p}=H(T, P)-$ $H(T, 0)=U+P / D-R T$

- The potential energy density $U D$ - the product of molar potential energy by density; - RTD

- The potential enthalpy density $H_{p} D=U D+P$

- The apparent equilibrium constants for different thermodynamics Excess functions series expansions by degrees of the monomer fraction density;

- The cluster bond energy $E_{n}$ - the distance from the monomers' free movement averaged energy to the thermodynamically averaged value of energetic levels in a cluster.

\subsection{The ideal gas laws applicability for real gases}

As we mentioned before, the clusters now are considered as a new, still unknown, state of matter. To study their nature and their role in gases is a challenging task for the thermodynamics education development. The real gas differs from an ideal gas by clusters' influence on its thermophysical properties, but may be considered as a mixture of ideal cluster fractions.

The ideal gas laws play an important role in pure real gases via the ideal laws for partial pressures of the monomer $P_{m}$ and $n$-particle cluster fractions $P_{n}$ :

$$
\begin{aligned}
& P_{m}=R T D_{m}, \\
& P_{n}=R T D_{n} .
\end{aligned}
$$

And the ideal gas equation for the total pressure:

$$
\begin{aligned}
& P=R T D_{p}, \\
& D_{p}=D_{m}+\sum D_{n}, n=2,3, \ldots
\end{aligned}
$$

The system of equations (1-4) is obvious, understandable, educative, and clearly explains the nature of pressure in real gases.

\subsection{The Monomer Fraction Density, $D_{m}$}

The Chemical Potential $G$ for all basic particles of a total gas is the same as for Monomers [15]. It looks like a universal Fermi level for free electrons and holes and for electrons on the donor and acceptor levels in semiconductors. The pressure dependent part of the Gibbs energy $G_{t r}$, as the translational part of $G$ for a real gas, is the same as $G_{\text {trm }}$ for an ideal gas of Monomers, Figure 2:

$$
G_{t r}=G_{t r m}=R T \ln \left(D_{m} V_{q}\right),
$$

where $V_{q}=h^{3} N_{A}^{4} /(2 \pi M R T)^{3 / 2}$ - the molar quantum volume [29], which is proportional to the third degree of the thermal de Broglie wavelength; $h$ is the Plank's constant, $M$ is the basic particles' molecular mass in $\mathrm{kg} / \mathrm{mol}, N_{A}$ is the Avogadro number, $R$ is the universal gas constant.

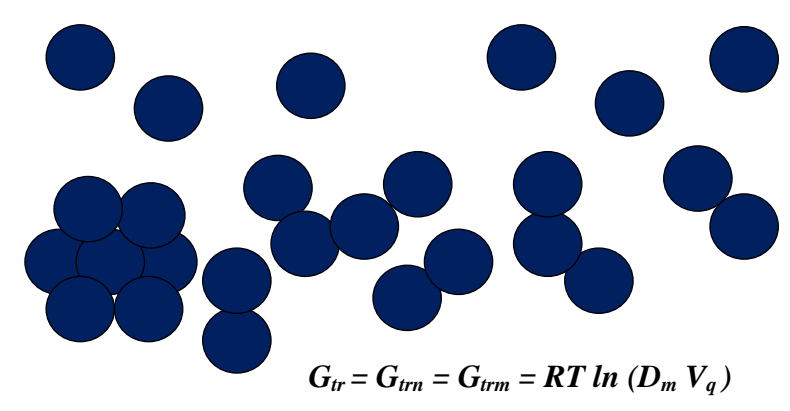

Figure 2. The translational part of the pure real gas basic particles' chemical potential $G$ independence on the structure of clusters.

For the total chemical potential $\mathrm{G}$ we have the wellknown thermodynamics law:

$$
\partial G /\left.\partial P\right|_{T}=V=1 / D
$$

Differentiating the $G_{t r}$ from equation (5) we come to the differential equation for $D_{m}[15]$ :

$$
\partial D_{m} /\left.\partial P\right|_{T}=D_{m} /(R T D)
$$


The equation (6) looks like the well-known equation for fugacity $f$ :

$$
\partial \ln (f) /\left.\partial P\right|_{T}=1 /(R T D)
$$

The fugacity $f$ had been introduced in thermodynamics by G. N. Lewis [30], but the monomer fraction density $D_{m}$ seems to be much more understandable for students. We can define the fugacity as the Monomer fraction partial pressure $P_{m}$ given by the equation (1).

The equation (6) can be solved numerically very precisely, if the initial $P_{l}$ and the pressure step values $\Delta P_{i}$ $=P_{i}-P_{(i-1)}$ are enough small. The online NIST Webbook [3] permits to download the isothermal thermophysical properties table for a selected pure gas with any desired pressure step value, thus providing a required precision of the $D_{m i}$ values computation. We recommend the next form of the equation (6) numerical solution [20]:

$$
D_{m i}=D_{m(i-1)}\left(1+\Delta D_{P i} /\left(2 D_{(i-1)}\right)\right) /\left(1-\Delta D_{P i} /\left(2 D_{i}\right)\right) .
$$

As an initial condition for an extra diluted gas we have: $D_{m_{1}}=2 D_{P_{1}}-D_{1}$. After $D_{m i}$ computation we add to the thermophysical properties table the $D_{m}$ i column, which may serve as an argument for series expansions of thermophysical functions.

It is difficult to define the monomer fraction density microscopically, because the boundary between the monomers' free movement and their bound states is vague. But the phenomenological approach via equation (6) permits to define the monomer fraction density in a real gas, and this definition may be used to test and correct the molecular simulation models.

The need in densities, $D, D_{p}$ and $D_{m}$, characterizing the pure real gas system, is clearly seen from the Figure 3, which shows that in the Carbon dioxide real gas at a supercritical temperature and at pressures higher than 20 bar all three densities differ very much. We use here the 'bar' unit for pressure, because this unit is close to the widely used in books and practice 'atmosphere' unit.

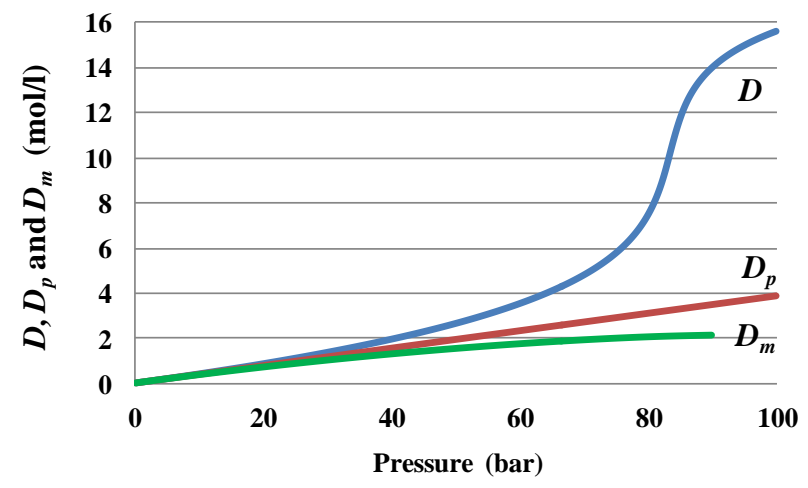

Figure 3. Densities: $D, D_{p}$ and $D_{m}$ for $\mathrm{CO}_{2}$ at a supercritical temperature, $T=310 \mathrm{~K}$.

The Figure 3 shows the $D_{p}$ graph going between the $D$ and $D_{m}$ lines. It means that the coefficients for $D_{p}$ series expansion by $D$ and $D_{m}$ have opposite signs. Indeed, the $C_{2}$ (T) coefficient of the $D_{p}$ series expansion by $D_{m}$ equals to the second virial coefficient $B(T)$ with an opposite sign: $C_{2}(T)=-B(T)$. A large difference between $D$ and $D_{m}$ results in a big difference between virial expansion of $D_{p}$ by degrees of $D$ and canonical expansion of $D_{p}$ by degrees of $D_{m}$. The Figure 3 shows that at the density $D$ near the critical density $D_{c r}$, which for $\mathrm{CO}_{2}$ is close to $10,6 \mathrm{~mol} / \mathrm{l}$, the curvature of the $D(P)$ line changes its sign, thus reflecting the gas-to-liquid transfer in a supercritical fluid. But the $D_{p}(P)$ and $D_{m}(P)$ curves are not sensitive to this transformation.

\subsection{The entropy of the pure molecular real gas}

The pure molecular real gas entropy $S$ may be split on:

- The pressure independent part for internal molecular vibrations and rotations, $S_{\text {int }}(T)$;

- The pressure dependent part for molecular and clusters' translational movements entropy, $S_{t r}(T, P)=S(T$, $P)-S_{\text {int }}(T)$.

From the equation (5) we come to the pressure dependent part of entropy $S_{t r}(T, P)[15]$ :

$$
S_{t r}=H_{t r} / T-R \ln \left(D_{m} V_{q}\right) .
$$

This equation for molecular real gases is the generalization of the well-known Sackur-Tetrode equation [31-33] for an atomic ideal gas:

$$
S=2.5 R+R \ln \left(V / V_{q}\right)
$$

It is useful for students to apply the equation (9) for different pure real gases with known precise thermophysical properties.

\subsection{The Apparent Equilibrium Constants for $n$ - particle Complexes}

The impossibility to find the cluster fractions' concentrations by direct physical methods forces us to use series expansions of experimental thermophysical functions to estimate different properties of the cluster fractions. Thus we come to a multitude of apparent equilibrium constants corresponding to different thermophysical functions.

These apparent equilibrium constants may look very strange. So, the coefficients $C_{n}(T)$ of the $D_{p}$ series expansion by $D_{m}$ change their sign at some temperatures. For example, the dimers' apparent equilibrium constant $C_{2}$ $(T)$, which equals to $-B(T)$, changes its sign at the Boyle temperature.

The coefficients $C_{n}(T)$ correspond to the $n$-particle complexes in agreement with the law of Mass action [16]. They may be named as apparent equilibrium constants for these complexes. But the $n$-particle complexes include diversified isomer structures of $n$-particle clusters, shown at Figures 1 and 2, which have different bond energies due to different numbers of bonds between particles. For this reason the temperature dependence of coefficients $C_{n}(T)$ is more complex than the Boltzmann law for chemical compounds.

Moreover, the collisions between monomers and small clusters in $n$-particle complexes do not always result in the $n$-particle cluster formation. Collisions followed by flying away of the colliding particles limit the free movement volume for them, thus introducing negative terms in the $C_{n}$ (T) values. It explains the sign changes of the $C_{n}(T)$ values at high temperatures.

\subsection{The real and virtual clusters}


Now we consider the structure of the free moving particles density $D_{p}=P / R T$. The free moving particles are monomers $[14,15]$, clusters $[17,18]$ and the arising at collisions temoparily existing complexes of monomers and clusters, which do not result in the clusters formation. These complexes may be named as virtual clusters [15], Figure 4. They limit the free movement volumes for monomers and clusters and manifest themselves through the excluded volumes.

Their contribution in thermophysical properties of gases also corresponds to the Mass action law.
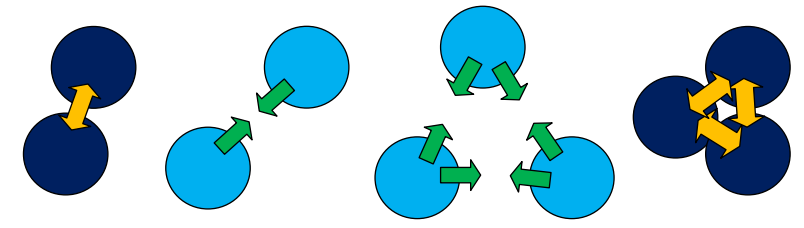

Figure 4. Real (dark balls) and virtual (light balls) clusters in dense gases: orange arrows - the bonds between particles in clusters; green arrows - the free moving paticles' moment components before collision.

In the chemical media the virtual clusters' contribution to the equilibrium constants of compounds is negligible, but in real gases the molecular interaction forces are much weaker, and the contributions of real and virtual clusters in the series expansion coefficients are comparable. Due to the virtual clusters existence the second virial and canonical coefficients change their sign at the Boyle temperature.

\section{The canonical cluster expansions}

\subsection{The $D$ and $D_{p}$ canonical expansions by degrees of} $D_{m}$

The series expansion of $D_{p}$ by degrees of $D_{m}$ at a constant $T$ may be written in a form:

$$
D_{p}=\Sigma C_{n} D_{m}^{n}, \quad n=1,2,3, \ldots \quad C_{1}=1 .
$$

Then, the equations (6) and (10) lead to the series expansion for $D$ :

$$
D=\sum n C_{n} D_{m}{ }^{n}, \quad n=1,2,3, \ldots \quad C_{1}=1 .
$$

The system of equations $(10,11)$ demonstrates an obvious relation between coefficients for $D$ and $D_{p}$ : the factor $n$ reflects the number of particles in the $n$-particle cluster.

The difference $D-D_{p}$ removes the monomers' contribution and thus helps to find the $C_{n}(T)$ values:

$$
D-D_{p}=\Sigma(n-1) C_{n} D_{m}{ }^{n}, \quad n=2,3, \ldots
$$

We expand in a series the function $C_{2+}=\left(D-D_{p}\right) / D_{m}{ }^{2}$. The limit of this function at zero $D_{m}$ value gives us the second canonical coefficient $C_{2}$. The third canonical coefficient $C_{3}$ we find as the zero $D_{m}$ limit of the function $C_{3+}=\left(C_{2+}-C_{2}\right) / 2 D_{m}$. And so on. The computation of these limits requires taking measures against errors in the $C_{n+}$ values at small $D_{m}$. The interactive process of coefficients $C_{n}$ computation permits to estimate and minimize the arising errors.
The Figure 5 shows the temperature dependence of the second apparent equilibrium constant $C_{2}(T)$ for Carbon dioxide. And the Figure 6 shows $C_{3}(T)$.

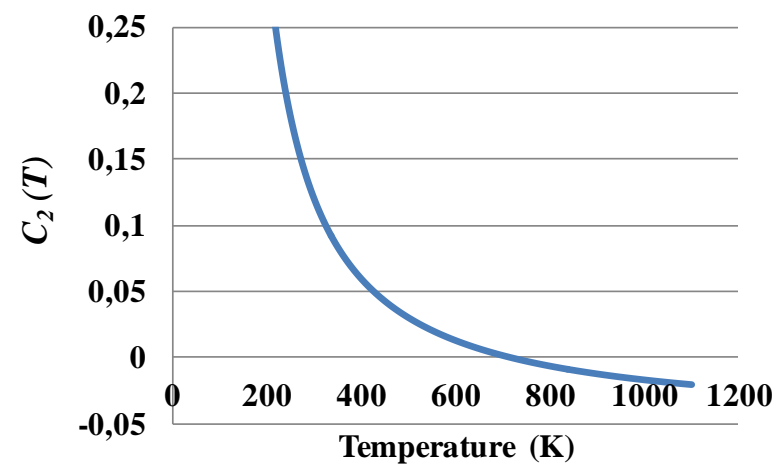

Figure 5. The second apparent equilibrium constant $C_{2}(T)$ for Carbon dioxide changing its sign at $T=722 \mathrm{~K}$ due to the virtual dimers' contribution.

It is seen that $C_{2}(T)$ becomes negative over the Boyle point $T=722 \mathrm{~K}$. This change of sign is due to a combination of two interaction mechanisms: the attraction and repulsion between colliding particles. We do not pay attention to this cooperation in chemical reactions because the chemical attraction forces are usually much stronger than the repulsion forces in gases. But, the investigation of aggregates with weak chemical bonding, such as the dimers in alkali metal vapors [34], may show the repulsions' negative contribution to their equilibrium constants.

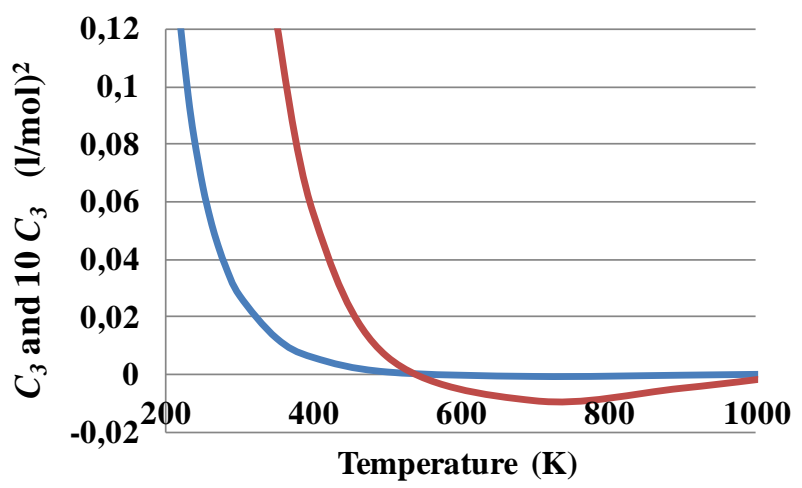

Figure 6. The third apparent equilibrium constant $C_{3}(T)$ for Carbon dioxide (blue line) and $10 C_{3}(T)$ (red line).

It is seen that the $C_{3}(T)$ changes its sign at $\mathrm{T}=578 \mathrm{~K}$. For many pure real gases the third apparent equilibrium constant $C_{3}(T)$ is almost equal to the double second power of the $C_{2}(T): C_{3}(T) \sim 2 C_{2}(T)^{2}$, Figure 7 . Here the negative part of $C_{3}(T)$ may be considered as zero. 


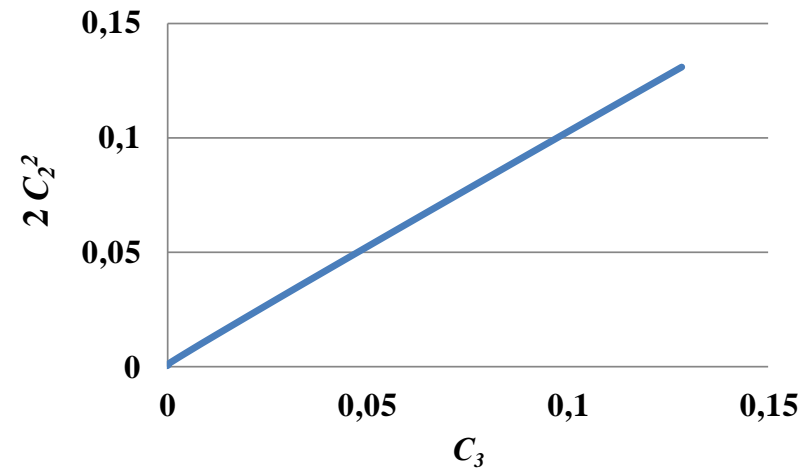

Figure 7. A comparison of the $2 C_{2}(T)^{2}$ with $C_{3}(T)$ for Carbon dioxide, demonstrating their proximity.

This proximity has a clear physical sense: the trimer with two bonds, shown at the Figure 1, may be considered as a complex of two dimers, in which the third particle may be attached to both ends of the existing dimer. It gives the factor 2 in the expression $2 C_{2}(T)^{2}$. The correlation of these apparent equilibrium constants tells in favor of the canonical cluster expansion, which provides a clear picture of small cluster fractions.

To estimate the contributions of different mechanisms of molecular interactions in the clusters' properties we should not limit our research by the pressure-densitytemperature (PDT) analysis, but to involve also the potential energy (PE) analysis [17].

\subsection{Coming to Clusters' bond parameters via Potential Energy}

The pure real gas potential energy [17] presents one of Excess thermodynamics functions, which expresses the difference between the real and ideal gases' Internal energies. Similar Excess thermodynamics functions may be introduced for Enthalpy, viscosity, thermal conductivity, and so on. They help to discover the pure real gas nature. The potential energy canonical cluster expansion has the next steps [17]:

- $\quad$ Molar Potential Energy of a fluid $U(T, P)$ is the difference of the Internal Energy $E(T, P)$ and its zero pressure value: $U(T, P)=E(T, P)-E(T, O)$.

- $\quad$ The Potential Energy density is $U D$.

- We expand in a series by $D_{m}$ a positive function:

$$
W_{2+}=-U D / D_{m}^{2} \text {. }
$$

- The series expansion coefficients $W_{n}$ provide the bond parameters and equilibrium constants of clusters discovery $[17,35]$.

- The coefficients $W_{n}$ are products of the apparent equilibrium constants $C_{u n}$ for the potential energy density and the clusters' bond energies $E_{n}$.

- The cluster bond energy $E_{n}$ in Kelvin can be found as:

$$
E_{n}=-T^{2} d \ln \left(W_{n}\right) / d T
$$

The Figure 8 shows the soft structural transition in the Water vapor tetramers from densely packed isomers dominating at low temperatures to the loosely packed isomers dominating at high temperatures $[35,36]$.

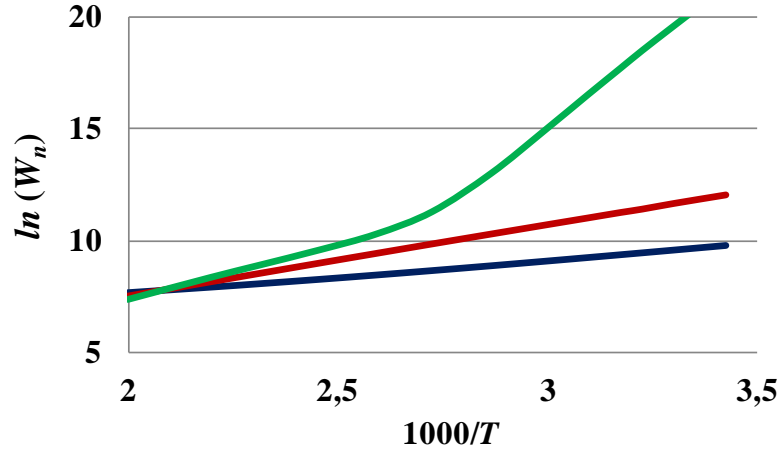

Figure 8. The soft structural transition between loosely bound isomers dominating at high temperatures and densely bound isomers dominating at low temperatures in the Water vapor tetramers: green line - tetramers; red line - trimers; blue line - dimers.

The Table 2 shows Water vapor clusters' bond energies found by the PE canonical expansion.

Table 2. Water vapor clusters' bond energies, measured in kilo Kelvin $(k K)$.

\begin{tabular}{|c|c|c|c|}
\hline Clusters & Dimers & Trimers & Tetramers \\
\hline$n$ & 2 & 3 & 4 \\
\hline$E_{n}(\mathrm{kK})$ at & 1,53 & 3,17 & 14,95 \\
$T<330 \mathrm{~K}$ & & & \\
\hline$E_{n}(\mathrm{kK})$ at & 1,53 & 3,17 & 4,9 \\
$T>400 \mathrm{~K}$ & & & \\
\hline
\end{tabular}

It is seen that at $\mathrm{T}>400 \mathrm{~K}$ the clusters bond energies are close to $(n-1) E_{2}$ that confirms the chain structure of clusters with the bonds number $N_{b n}=(n-1)$, but at $\mathrm{T}<$ $330 \mathrm{~K}$ the tetramers' bond energy is much higher. The three-fold growth of the $E_{4}$ cannot be explained by the growing number of bonds between four $\mathrm{H}_{2} \mathrm{O}$ molecules in the tetramer.

So, this effect tells about a stronger interaction between molecules in the Water vapor tetramers. The gases, like the Water vapor, with this type of the soft structural transition in their cluster fractions are not very multiple. The most of gases, such as the Argon, Nitrogen, Carbon Dioxide, do not have this type of a soft structural transition and may be named as normal.

\subsection{The virtual clusters}

The virtual clusters appear in real gases in three forms: 1. The basic particles repulsion volume, $V_{e x}$, which limits the free movement volume for a basic particle due to its collisions with other particles;

2 . The monomer-cluster interaction volume, $V_{\text {int }}$, which should be excluded from the monomer free movement volume to avoid the double counting of this volume for clusters and for monomers.

3. The elastic collisions volume $V_{e c}=-T d V_{e x} / d T$. The collisions' elasticity contributes to the potential energy of a real gas and changes the corresponding apparent equilibrium constant [37].

The most understandable are virtual dimers in atomic gases. All three volumes, responsible for virtual dimers, are shown schematically at the Figure 9. 


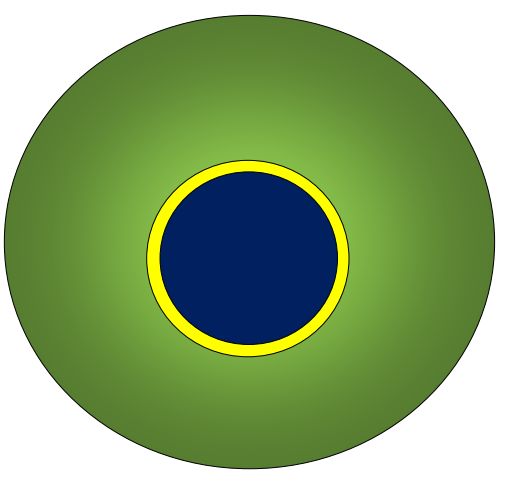

Figure 9. Three volumes responsible for virtual dimers in atomic real gases: the excluded volume $V_{e x}$-blue ball; the elastic collision volume $V_{e c}$ - yellow sphere; the monomerdimer interaction volume $V_{\text {int }}$-green sphere.

The zones of three volumes are determined by the pair interaction energy dependence on the distance $d$ between atoms, Figure 10. For simplicity we use here the LennardJones interaction model potential [38]. For example, the excluded volume $V_{e x}=2 \pi N_{a v} d^{3} / 3$. Here $N_{a v}$ is the Avogadro number. The change of the factor 4 in a standard formula for volume on 2 accounts for two particles dividing their common excluded volume.

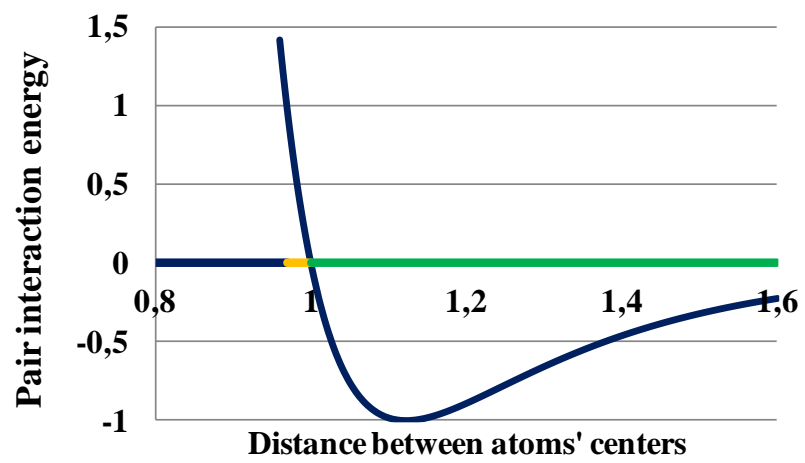

Figure 10. The three distance zones for three volumes calculation at a temperature $T$ equal to the maximal depth of the potential well: the excluded distance marked by the blue horizontal line, starting at a zero distance; the elastic collision distance marked by the yellow line; the dimers' attraction distance marked by the green line.

From the Figure 10 it is seen that the distance between colliding particles depends on the collision energy and the elasticity of this dependence determines the elastic collision volume $V_{e c}=-T d V_{e x} / d T$.

To find the pair interaction bond energy $E_{2}$ we have to account for the monomers elastic collisions contribution into total potential energy of a gas. This contribution to $W_{2}$ grows with temperature almost linearly: $\Delta W_{2}=R T V_{e c}$ [37]. To correct the $W_{2}(T)$ from the monomers elastic collisions contribution into total potential energy we use the corrected function $W_{2 c o r}(T)=W_{2}(T)+R T V_{e c}$ and find the fitting $V_{e c}$ value with a criterion: the calculated temperature dependence of the $V_{\text {int }}(T)$ at the highest temperatures in the database should be flat. The Figure 11 shows the corrected $\mathrm{CO}_{2}$ dimers bond energy with $d V_{\text {int }} /$ $d T=0$ at $T=1100 \mathrm{~K}$.

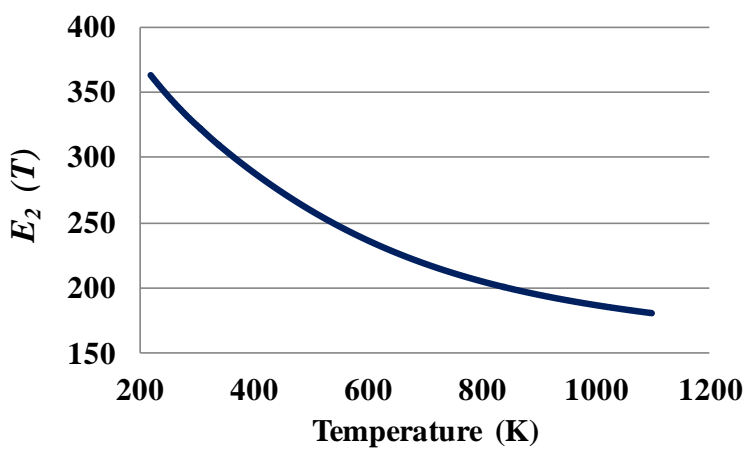

Figure 11. The corrected $\mathrm{CO}_{2}$ dimers bond energy $E_{2}(T)$ demonstrating the soft transition from the free rotation of molecules in dimers at high temperatures to a more oriented their position near the triple point

In the noble gases even atomic dimers at low temperatures exhibit a no spherical symmetry of their bonds due to a quantum character of interatomic forces and change their bond energy with temperature, Figure 12, towards freezing of the dimer structure near the triple point. It should be taken into account at the computer simulation of the noble gases' thermophysical properties.

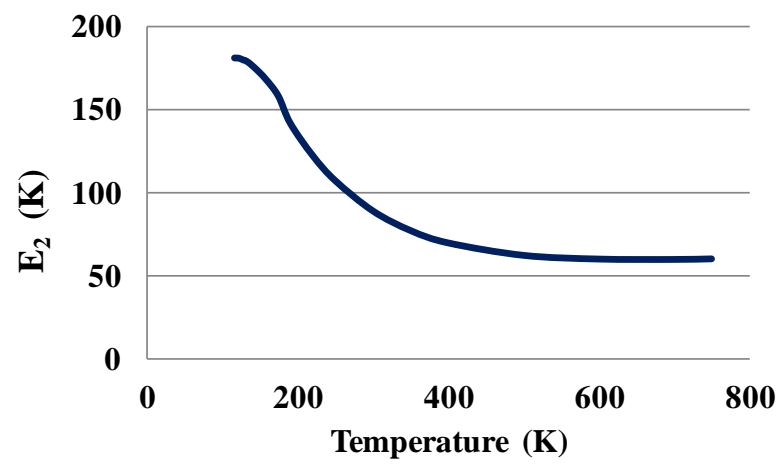

Figure 12. The pair bond energy $E_{2}(T)$ in Krypton, demonstrating freezing of the dimer structure near the triple point temperature.

The Figure 12 shows that near the triple point temperature, $T_{t r}=115.775 \mathrm{~K}$, in atomic gas Krypton the pair bond energy is three times larger than at temperatures over $500 \mathrm{~K}$ [36]. It may be explained as the soft structural transition from spherical symmetry of interatomic bonds at high temperatures to strongly oriented bonds [37] inherent to the solid state of Krypton.

\section{A symmetry between clusters in vapors and pores in liquids at a thermal equilibrium}

For many pure fluids the sum of vapor and liquid saturation densities has proven to depend linearly on temperature, Figure 13. It means the growth of the vapor saturation density with temperature is totally compensated in the sum by growing volume of pores in the saturated liquid. The linear change of the total density may reflect the special thermal expansion law for an infinite cluster, being free of pores. 


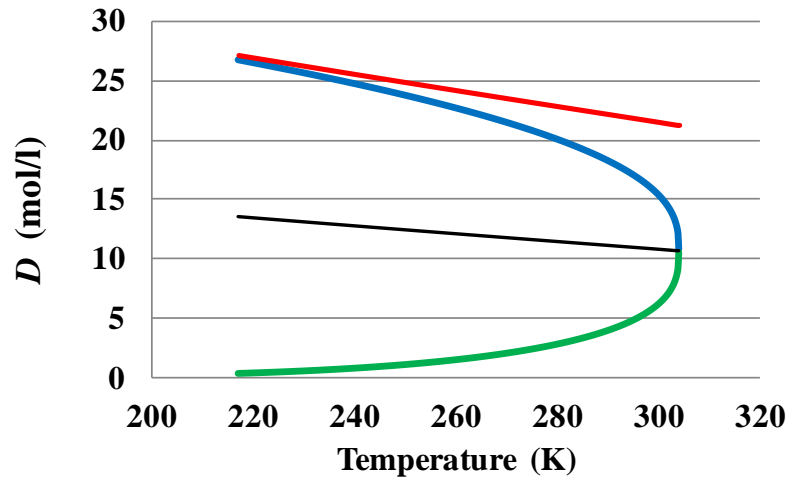

Figure 13. The linear temperature dependence of the sum of the $\mathrm{CO}_{2}$ saturation densities (red line): the vapor density (green line); the liquid density (blue line); the mirror density, equal to a half of the sum of densities (black line), pointing out to the critical density.

The sum of saturation densities may be attributed to the density of an infinite cluster, being free from pores. The difference between the infinite cluster density and the liquid saturation density reflects the effect of pores on the liquid saturation density. This effect seems to be equal to the vapor saturation density. At the critical temperature $T_{c}$ the critical density $D_{c}$ equals to a half of the infinite cluster critical density. The line corresponding to density, which equals to a half of the infinite cluster density is situated exactly on equal distances between the saturation densities and for this reason may be named as the mirror line.

So, for many fluids between microscopic structures of pores in liquids and clusters in vapors along the saturation line may exist a statistical symmetry [39] depicted on the Figure 14.
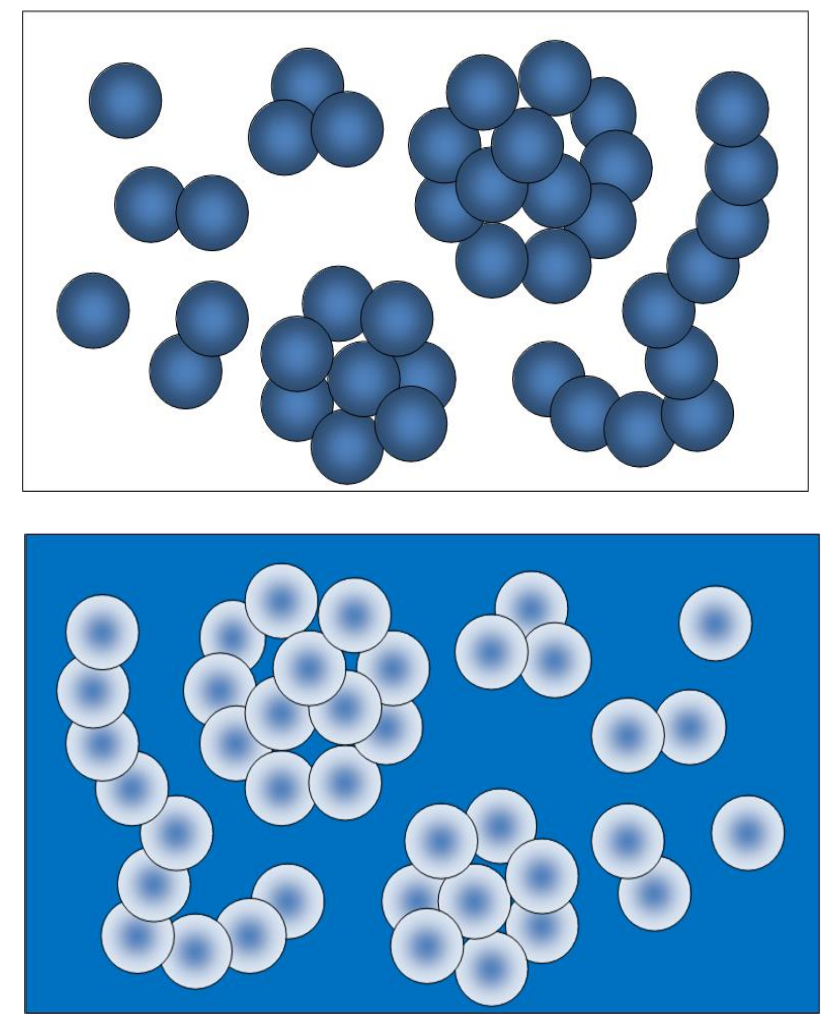

Figure 14. The statistical symmetry between cluster fractions in the vapor (upper picture) and pores fractions in the liquid (lower picture) at a vapor-liquid equilibrium.
5. Step-by-step movement from ideal to dense real gases

Moving step-by-step from ideal to denser gases we approach to microscopic structure of the condensed matter. On this way we have discovered [40] the linear chain clusters at the density range $D<D_{c r} / 5$. In this range of densities the isothermal dependences of the $1 / C_{2+}$ and $1 / W_{2+}$ on $D_{m}$ are linear for many pure real gases. This linearity tells about existence of linear chain clusters with no more than two bonds for a particle.

In the density range over $D_{c r} / 5$ and up to $D_{c r}$ together with linear chain clusters appear large three dimensional (3D) clusters [41]. To study characteristics of these clusters a special logarithmic expansion method has been developed. This method permits the clusters' properties estimation for numbers of particles in them up to 1 thousand. These huge clusters at the critical density and supercritical temperature merge into an infinite cluster filled with pores.

The interpretation of these wonderful phenomena will be given in the next part of the paper devoted to dense gases.

\section{Conclusions}

- The unique feature of pure real gases, the uniformity of the basic particles' Chemical Potential for clusters and the total gas, provides the clusters' properties cognition.

- $\quad$ The Monomer Fraction Density, as a key variable in the Thermodynamics of pure real gases, supports discovering of their cluster structure.

- The Monomer Fraction Density permits the Sackur-Tetrode equation generalization to molecular real gases.

- The Potential Energy based analysis of precise equilibrium thermophysical data delivers the clusters' structural and bond parameters.

- The temperature dependences of the clusters' bond energies reveal the soft structural transitions between cluster isomers.

- $\quad$ All new phenomena in pure real gases make this platform interesting for students and motivate them to new investigations and discoveries.

\section{Nomenclature}

MFD Monomer Fraction Density

$D \quad$ Molar density of basic particles, $\mathrm{mol} / \mathrm{l}$

$P \quad$ Pressure, bar

$n \quad$ Number of particles in the cluster and the order of the series expansion term

$D_{n} \quad$ Partial molar density of the $n$-order cluster fraction, $\mathrm{mol} / \mathrm{l}$

$D_{m} \quad$ Monomer fraction density, mol/l

$P_{n} \quad$ Partial pressure of the $n$-order cluster fraction, bar

$C_{n}(T)$ Apparent equilibrium $n$-order cluster formation constant, reflecting both attraction and repulsion forces

$C_{u n}(T)$ Apparent equilibrium $n$-order cluster formation constant, found from potential energy density

E Molar internal energy, $\mathrm{J} / \mathrm{mol}$

$U \quad$ Molar potential energy, $\mathrm{J} / \mathrm{mol}$

$H \quad$ Molar enthalpy, $\mathrm{J} / \mathrm{mol}$

$G \quad$ Molar Gibbs energy (Chemical potential), J/mol

$G_{t r} \quad$ Translational part of the molar Gibbs energy 
$G_{\text {int }} \quad$ Intramolecular part of the molar Gibbs energy

$S \quad$ Molar entropy, $J /(\mathrm{mol} \mathrm{K})$

$S_{\text {int }} \quad$ Intramolecular rotations and vibrations entropy

$S_{t r} \quad$ Translational part of the entropy, $J /(\mathrm{mol} \mathrm{K})$

$V_{i} \quad$ Volume for a mole of an ideal gas, $l / \mathrm{mol}$

$V_{q} \quad$ Molar quantum volume for basic particles, $\mathrm{l} / \mathrm{mol}$

$h \quad$ Plank's constant

$M \quad$ Molecular weight, $\mathrm{kg} / \mathrm{mol}$

$N_{A} \quad$ Avogadro number, $1 / \mathrm{mol}$

$R \quad$ Universal gas constant, $J /(\mathrm{mol} \mathrm{K})$

$W_{n} \quad$ Apparent equilibrium $n$-order constant for potential energy density

$E_{n} \quad$ Bond energy for $n$-particle cluster, $K$

$V_{e x} \quad$ Excluded volume for pair interactions, $l / m o l$

$V_{e c} \quad$ Pair elastic collision volume, $l / \mathrm{mol}$

$V_{\text {int }} \quad$ Pair interaction volume in a dimer, $l / m o l$

\section{References:}

[1] Kaplan I.G., Intermolecular Interactions: Physical Picture, Computational Methods and Model Potentials New York: Wiley - Interscience, 2006.

[2] Rowlinson, J.S., Cohesion: A Scientific History of Intermolecular Forces, Cambridge: Cambridge University Press, 2002.

[3] NIST Webbook: Thermophysical Properties of Fluid Systems. 2011. [Online]. Available: https://webbook.nist.gov/chemistry/fluid/ (accessed: Dec. 21, 2019).

[4] Yarris L. "Clusters: A New State of Matter," Berkeley LAB Publication Archive, 1991. Available: https://www2.lbl.gov/Science-

Articles/Archive/clusters.html (accessed Dec. 22, 2019).

[5] Gas-Phase Synthesis of Nanoparticles. Ed. Y. Huttel, Wiley Online Books, 2017 Available: https://onlinelibrary.wiley.com/doi/book/10.../9783527698 $4 \ldots$

[6] Huttel Y., Gas Aggregation Synthesis of Nanoparticles, Wiley-VCH, 2017, 416 p.

[7] Smirnov B.M. Cluster Processes in Gases and Plasmas, Wiley-VCH, 2010.

[8] De Micheli S.M., Licenblat A.R. "Ice Whiskers Grown at Subsaturated Atmospheres," J. Atmospheric Sciences, 24, 1967, 312-315.

[9] M. Türk. Particle Formation with Supercritical Fluids, Volume 6 in the Series: Supercritical Fluid Science and Technology, Editor - Erdogan Kiran, Elsevier, 2014.

[10] Acharya A., Nayak R. L., "Alpha clustering study in even nuclei," Int. J. of Current Research, 2016, 8 (03), 27401-27406.

[11] Kusaka, I., Oxtoby, D.W., "Identifying Physical Clusters in Vapor Phase Nucleation", J. Chem. Phys. 1999, 110 (20), 5249.

[12] Harris, S.S., Ford, I.J., "A Dynamical Definition of Quasibound Molecular Clusters", J. Chem. Phys., 2003, 118 (20), 9216.
[13] Ford, I.J., "Statistical mechanics of nucleation: a review", Proc. Instn. Mech. Engrs., J. Mechanical Engineering Science, 2004, 218 (C), 883.

[14] Sedunov, B., "Monomolecular fraction in real gases", Proceedings of Joint European Thermodynamics Conference, JETC IX, 2007, 176-180.

[15] Sedunov B. "Monomer fraction in real gases," Int. J. of Thermodynamics, 2008. 11 (1) 1-9.

[16] Koudryavtsev, A.B., Jameson, R.F. and Linert, W. The Law of Mass Action, 2001, Berlin: Springer-Verlag.

[17] Sedunov B. "Equilibrium molecular interactions in pure gases," J. of Thermodynamics, 2012. - Article ID 859047. - 13 pages. DOI: 10.1155/2012/859047

[18] Sedunov B. "Equilibrium Structure of Dense Gases," Proc. of the JEEP-2013, Nancy: MATEC Web of Conferences. - DOI:10.1051/matecconf/20130301002

[19] Aster R.C., Borchers B., and Thurber C. Parameter Estimation and Inverse Problems, 2nd edn. Elsevier. 2012.

[20] Sedunov, B., "New approaches to teaching the Thermal Physics of Fluids," 21-st IUPAC International Conference on Chemical Thermodynamics ET-5101-1540, Tsukuba, Japan, 2010

[21] B. Berche, M. Henkel, R. Kenna. "Critical Phenomena: 150 Years Since Cagniard de la Tour," J. of Physical Studies, 13( 3) 2009, 3001

[22] T. Andrews. "The Bakerian Lecture.- On the Continuity of the Gaseous and Liquid States of Matter," 1869.

[23] T. Andrews, Phil. Trans. R. Soc. London, 159, 1869, 575.

[24] T. Andrews, Phil. Trans. R. Soc. London, 166, 1876, 421.

[25] Mayer, J.E. and Goeppert-Mayer, M. Statistical Mechanics, 2nd Ed. New York: Wiley \& Sons. 1977.

[26] Mayer J.E., "Contribution to Statistical Mechanics," J. Chem. Phys. 10, 1942, 629-643.

[27] Ford I.J. "Virial/Fisher Models of Molecular Cluster Populations" J. Chem. Phys. 1997, 106 (23), 9734.

[28] Feynman, R., Statistical mechanics; A set of lectures, Massachusetts: Benjamin, Inc., 1972, 115-147.

[29] Kittel, Ch., Thermal physics, New York: John Wiley and Sons, Inc., 1969.

[30] G.N. Lewis and M. Randall, Thermodynamics and the Free Energy of Chemical Substances, New York: McGraw-Hill Book Company, 1923.

[31] Tetrode, H., 1912, Ann. der Physik, 38, 434.

[32] Sackur, O., 1913, Ann. der Physik, 40, 67.

[33] Grimus W., "On the 100th anniversary of the SackurTetrode equation", arXiv:1112.3748v2 [physics.hist-ph] 23 Jan 2013. 
[34] Wargaftik N.B. Handbook of Physical Properties of Liquids and Gases: Pure Substances and Mixtures. Berlin-Heidelberg: Springer-Verlag, 1975.

[35] Sedunov B. Discovering the Cluster World. Saarbrucken, Germany: Lambert Academic Publisher. 2015, 102 p.

[36] Boris Sedunov. The Monomer Fraction Density Based Theory of Real Gases. JETC_book_of_abstracts. 2019, p. 109.

[37] Sedunov B. The Wonders of Molecular Interactions. Saarbrucken, Germany: Lambert Academic Publisher. 2016, 98 p.

[38] Lennard-Jones, J. E., "On the Determination of Molecular Fields", Proc. R. Soc. Lond. A, 1924, 106 (738): 463-477.

[39] Boris Sedunov. "Thermodynamics of the Pure Real Gas System," JETC_book_of_abstracts. Barcelona, 2019, 72.

[40] Sedunov B. "The Analysis of the Equilibrium Cluster Structure in Supercritical Carbon Dioxide," American J. of Analytical Chemistry. 2012. - Issue 3 (12A). - pp. 899904. DOI: 10.4236/ajac.2012.312A119

[41] Sedunov B. "Nanosized objects in equilibrium supercritical fluids," Proc. of the JEEP-2013, Nancy: MATEC Web of Conferences. 3. - 01062. - 2013. DOI:10.1051/matecconf/20130301062 